\title{
Intermediate structures in two-dimensional molecular self-assembly
}

\author{
Karl-Heinz ERNST ${ }^{1,2}$ \\ ${ }^{1}$ Nanoscale Materials Science, Empa, Swiss Federal Laboratories for Materials Testing and Research, Ueberlandstr. 129, \\ CH-8600 Dübendorf, Switzerland \\ ${ }^{2}$ Fachbereich Chemie, Universität Zürich, CH-8057 Zürich, Switzerland \\ E-mail: Karl-Heinz.Ernst@empa.ch \\ Received May 28, 2010; accepted June 20, 2010
}

\begin{abstract}
We discuss the occurrence of transition structures observed in molecular self-assembly at surfaces. The increasing surface coverage transitions from low coverage structures to high coverage structures are a common phenomenon. However, often observed and not perfectly understood is the formation of intermediate structures, sometimes with lower lateral density than the initial phase. We will present different examples from our recent work and discuss the possible mechanisms of intermediate phase formation. In addition, we present intermediate structures occurring due to temperature-controlled reversible phase transitions.
\end{abstract}

Keywords scanning tunnelling microscopy (STM), polymorphism, corannulene, heptahelicene, chirality

PACS numbers 82.65.+r, 61.05.jd, 68.35.Ja, 61.43.-j, 64.75.Yz

\section{Contents}

1 Introduction

2 Temperature-induced intermediate phases

2.1 Reversible 2-D phase transitions

2.1.1 A thermodynamically stable intermediate

2.1.2 A metastable phase via transition blocking

2.2 A 2-D glass coexisting with ordered phases 342

3 Coverage-dependent intermediate phases

3.1 Azimuthal reorientation

3.1.1 Helical aromates

3.1.2 A bowl-shaped phthalocyanine

4 Conclusions

5 Methods and materials

Acknowledgements

References

\section{Introduction}

Polymorphism, the phenomenon that a single compound can appear in different crystal modifications, is of paramount importance in pharmaceutical and materials science. A new polymorph, for example, may act as contamination, inhibiting formation of other polymorphs in a laboratory forever [1]. This has been experienced with the anti-viral drug Ritonavir, which had to be withdrawn from the market after a new, less soluble crystal form appeared in a new synthesis batch [2]. Polymorph control is therefore critical for the success of a pharmaceutical product [3]. The mechanism of how one polymorph induces re-crystallization of another is not understood. Important steps include molecular stereochemical recognition at the crystal surface and a solid-state phase transition [4]. Solid-solid phase transitions and metastable polymorphs are common, making polymorph selection challenging. Nature teaches us, however, that control of polymorphism is possible, since many organisms "choose" shape, habit and polymorph of inorganic minerals in biomineralization [5].

It has been demonstrated that studies of well-defined model systems help to unravel details of surface reactions [6]. Consequently, the self-assembly of molecules on single crystal surfaces is increasingly deployed as an approach for better understanding of stereochemical recognition at surfaces [7]. In particular, scanning tunnelling microscopy (STM) allows monitoring these complicated processes at the molecular level [8].

\section{Temperature-induced intermediate phases}

Fivefold-symmetry is regularly observed in nature and 
chemistry, but is incompatible with the translational order of a classical crystal lattice [9]. There are strong indications that supercooled liquids and metallic glasses possess icosahedral short-range order [10], and this geometry might be equally important for the glass transition and the structure of liquids during the melting process [11]. But how does a liquid freeze if the geometry of its molecules prevents close packing into a regular crystal? Or how do such molecules arrange themselves on a surface when they adsorb from a solution or the gas phase? In order to realize a perfect tiling in a plane, fivefoldsymmetric shapes must be combined with other shapes $[12,13]$. However, exclusive $\mathrm{C}_{5 \mathrm{v}}$ symmetry cannot be maintained in a periodic tiling in two-dimensional (2-D) supramolecular structures [14].

\subsection{Reversible 2-D phase transitions}

\subsubsection{A thermodynamically stable intermediate}

We have studied the adsorption of corannulene $\left(\mathrm{C}_{20} \mathrm{H}_{10}\right)$ on the copper (110) and (111) surfaces [15-19]. Corannulene (1, Fig. 1) is a curved, $\mathrm{C}_{5 \mathrm{v}}$-symmetric aromatic molecule with one pentagonal ring centred among hexagonal rings, thus representing a segment of $\mathrm{C}_{60}$ buckminsterfullerene [20]. At room temperature on $\mathrm{Cu}(111)$ a regular $(40,04)^{\#}$ array is observed for the complete corannulene monolayer (Fig. 1). Upon cooling, the 2-D crystal undergoes two different phase transitions. At $225 \mathrm{~K}$ a denser $(40,37)$ lattice forms, followed by formation of a $(42,07)$ structure with further decreasing temperature. Each structure is stable in its respective temperature interval. This is confirmed by the reversibility of the transitions (Fig. 1): When the low-temperature phase is heated, the medium-temperature phase forms again and is then converted completely into the $(40,04)$ phase at room temperature. To our knowledge, this is the first observation of an enantiotropic phase transition in a 2-D organic molecular crystal. Both transitions show a hysteresis in temperature. The $(40,37)$ structure exists at temperatures between 200 and $250 \mathrm{~K}$. The highest temperature observed for the $(42,07)$ structure was 236 $\mathrm{K}$ upon cooling and $271 \mathrm{~K}$ while heating.

The molecules in all three structures are imaged asymmetrically. This indicates a substantial tilt of the molecular bowl with respect to the surface plane and agrees well with a geometry in which the molecules are oriented with one of the five C6 rings parallel to the surface. Density functional theory (DFT) actually predicts the hexagonal ring to be located above a threefold hollow site [17]. For the $(40,37)$ intermediate structure, however, the two molecules of the unit cell are located

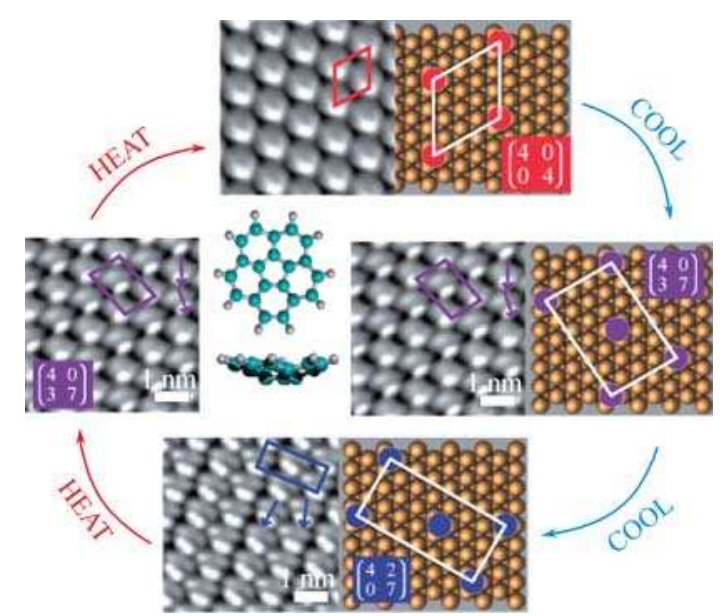

Fig. 1 Scheme of two reversible phase transitions between three polymorphs of $\mathbf{1}$ on $\mathrm{Cu}(111)$. STM images of the three observed phases (averaged from 186, 64, and 17 different positions, respectively). The unit cells and the molecular azimuthal orientations are indicated (scale bars are $1 \mathrm{~nm}$ ). A ball-and-stick model of $\mathbf{1}$, the unit cells on the copper grid and the matrices are also given. The intermediate $(40,37)$ phase is only observed between $200 \mathrm{~K}$ and $250 \mathrm{~K}$.

on different sites, i.e., one on an fcc threefold hollow site, the other on a hcp threefold hollow site. The longer unit cell vector runs at a small oblique angle with respect to the $\left\langle\begin{array}{lll}1 & 1 & \overline{2}\end{array}\right\rangle$ substrate directions (Fig. 1). Hence, the structure is enantiomorphous, i.e., reflection symmetry is broken and mirror-domains exist. Rotational domain boundaries therefore show a tilt angle for molecular rows as well (Fig. 2, inset). The two lower temperature phases are substantially denser $\left(1.26\right.$ molecules $\left./ \mathrm{nm}^{2}\right)$ than the room temperature phase (1.10 molecules $\left./ \mathrm{nm}^{2}\right)$, so the lattice density changes substantially during the (4 $0,04) /(40,37)$ transition, but not for the $(40,37)$ to $(42,07)$ transition. Without changing the number of molecules on the surface, the lattice density is increased by $14.5 \%$ during the first transition.

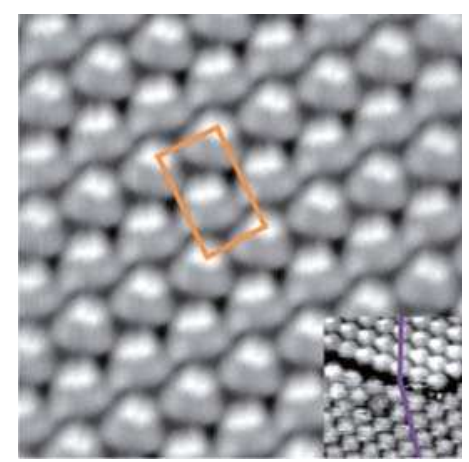

Fig. 2 STM image of the intermediate phase meta-stabilized at $67 \mathrm{~K}[10 \mathrm{~nm} \times 10 \mathrm{~nm}$, averaged over 59 positions $(-2 \mathrm{~V}, 1 \mathrm{nA})]$. The inset $(10 \mathrm{~nm} \times 10 \mathrm{~nm})$ shows a rotational domain boundary.

Attractive intermolecular interactions clearly drive the first transition upon cooling and increase the binding en-

\# The two-by-two transformation matrix $(m 11 \mathrm{~m} 12, \mathrm{~m} 21 \mathrm{m22})$ links the adsorbate lattice vectors $(b 1, b 2)$ to the substrate lattice vectors $(a 1, a 2)$ via: i.e., $b 1=m 11 a 1+m 12 a 2$ and $b 2=m 21 a 1+m 22 a 2)$ [23]. 
thalpy. Hence, the $(40,04)$ phase must have higher entropy due to vibrational excitations [21]. That excitation of vibrations at higher temperature lead to occupation of more space is well accepted and has been demonstrated recently for benzene [22]. Depopulation of vibrational states by means of cooling decreases the spatial requirements of the molecules and allows closer packing.

\subsubsection{A metastable phase via transition blocking}

The intermediate phase could be stabilized by soft breathing modes of the buckybowl as well. The driving force for the low-temperature (LT) phase transition upon cooling, however, is presumably the occupation of identical threefold hollow substrate sites in the LT phase, which are energetically slightly favoured. Although both phases have identical density, more space is needed during the transition. By lateral confinement with additional molecules, we succeeded to block this transition [18]. In that case the $(40,37)$ intermediate phase was stabilized down to $60 \mathrm{~K}$, a metastable structure at that temperature. This allowed, in turn, to achieve better resolved STM images (Fig. 2).

\subsection{A 2-D glass coexisting with ordered phases}

As a consequence of the contraction upon cooling, the bare copper surface must be revealed in other areas. Single molecules, too mobile to be imaged in STM, form a 2-D gas in these areas, but further cooling leads to 2-D solidification. Figure 3 shows STM images of the result. In part, no long-range order is observed and a 2-D glass of low density has been formed. Upon solidification of the 2-D gas, we also observe density fluctuations and locally mid-range ordering with higher density. That is, patches of ordered structures surrounded by the 2-D glass. Figure 3a shows such a new ordered phase embedded in the glassy area. This perfectly periodic structure contains different types of molecular orientations. Molecular bowls pointing upright are arranged into trimers $\left(\mathrm{C}_{3}\right.$ symmetry $)$, which are lined-up in rows. These rows are decorated with tilted molecules at the gaps between the trimers. In addition, located between these rows are ziczac rows of tilted molecules. Overall, there are seven molecules in this large unit cell. In areas with even higher density in the glass phase, structures with all molecules tilted into the same direction are observed again. Figure 3(b) shows an STM image of such ordered structure. Quite surprisingly, the lattice is almost quadratic, which is compatible with the underlying hexagonal metal substrate only in a $(43,-13)$ lattice. This shows that at low temperature $(74 \mathrm{~K})$ the locally self-assembled lattice due to solidification is slightly denser (equivalent to $15 \mathrm{Cu}$ atoms $)$ than the $(4 \times 4)$ lattice $(16 \mathrm{Cu}$ atoms $)$, which is observed at room temperature for the saturated monolayer.

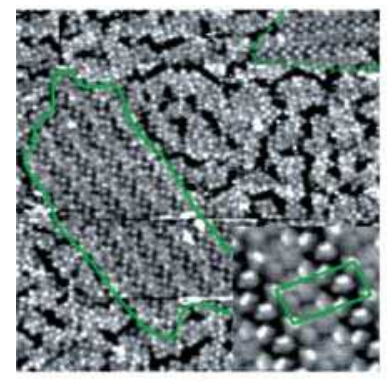

(a)

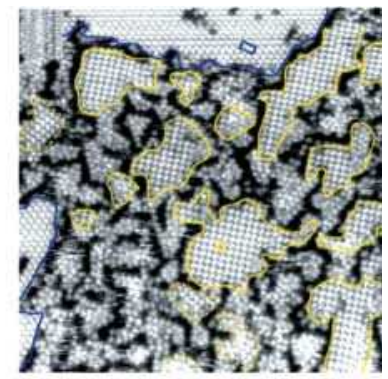

(b)
Fig. 3 (a) STM image $(50 \mathrm{~nm} \times 50 \mathrm{~nm})$ of an area showing an ordered structure within the glass. The inset shows an averaged seven-nm-image [parameters: -2000 mV, $200 \mathrm{pA}, 1149 \mathrm{~nm} / \mathrm{s}, 72 \mathrm{~K}$, (inset) is averaged over 17 positions]. (b) STM image (50 nm $\times$ $50 \mathrm{~nm}$ ) of a rectangular structure with medium density. Domains of this structure are marked yellow. The analysis shows a (34, -3 1) matrix and hence a local coverage of $1 / 15$ molecules per $\mathrm{Cu}$ atom. The blue lines mark the denser LT phase (parameters: $-1800 \mathrm{mV}, 50 \mathrm{pA}, 781 \mathrm{~nm} / \mathrm{s}, 66 \mathrm{~K})$.

Formation of these structures is explained by diffusion kinetics. At the low density of the 2-D gas, these low-density structures nucleate, but they are not able to grow further due to the limited diffusion with falling temperature. For the same reason, the LT phase does not completely dominate at low temperatures and the 2-D glass appears.

\section{Coverage-dependent intermediate phases}

\subsection{Azimuthal reorientation}

\subsubsection{Helical aromates}

2-D crystallization of the chiral aromatic hydrocarbon heptahelicene [Fig. 4(a) top, $\mathrm{C}_{30} \mathrm{H}_{18},[$ [7] $\mathrm{H}$ ] on metal surfaces is an exciting chapter of surface chirality. While on nickel surfaces no extended ordering phenomena has been found [24-26], a pronounced transfer of chirality into mono-and multilayered structures, manifested by the formation of extended chiral motifs, was observed for the pure enantiomers on $\mathrm{Cu}(111)[27,28]$. Interestingly, mirror domains have been observed for the racemate at the same surface, indicating at a first glance a lateral resolution of the enantiomers [29]. However, STM experiments in combination with molecular modeling calculations (MMC) showed that heterochiral M-P pairs become aligned in a chiral zigzag configuration (Fig. 4), leading to the observed enantiomorphism [30]. A switch to the opposite enantiomorph requires only a small rearrangement of the enantiomers in the heterochiral pair. This leads to the induction of lattice homochirality via cooperative amplification of a small chiral bias in nonracemic mixtures [30]. This kind of amplification of chirality is quite similar to the "sergeants-and-soldiers" 
principle, first reported for helical polymers with chiral side chains in solution [31]. In two dimensions this phenomenon has been first observed by us for racemic monolayers of prochiral succinic and meso-tartaric acid after doping with intrinsically chiral $(R, R)$-or $(S, S)$-tartaric acid [32-34]. The corresponding amplification of chirality in helical copolymers with small enantiomeric excess in the side chains has been coined as "majority rule" [35], and the 2-D analogue has been reported recently [36].

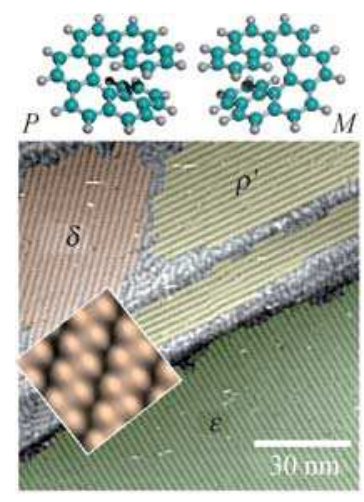

(a)

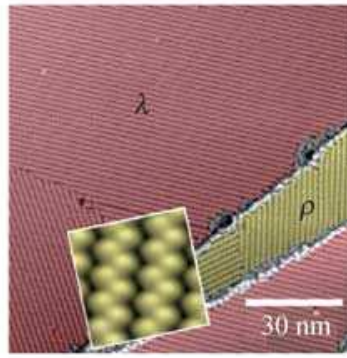

(b)
Fig. 4 Helicene polymorphs. (a) Top: Ball-and-stick model of the two [7] $\mathrm{H}$ enantiomers. Bottom: STM image of [7] $\mathrm{H}$ phases at medium coverage. The $\lambda^{\prime} / \rho^{\prime}$ phase (only the $\rho^{\prime}$ enantiomorph is shown) is an intermediate between the $\varepsilon / \delta$ phase and the $\lambda / \rho$ phase at saturation coverage (b). At lower coverage only the domains of the $\varepsilon / \delta$ phase are observed. The insets show the zigzag motif for the $\delta$ structure [(a) Bottom] and for the $\rho$ tructure (b).

No ordered superstructures are observed up to coverages close to half a monolayer. Even at $50 \mathrm{~K}$, single molecules are too mobile to be imaged via STM on the flat $\mathrm{Cu}(111)$ terraces. Hence, the diffusion barrier for the individual molecule is either low enough to allow the molecules to diffuse to the step edges where they get pinned, or they are moved by the STM tip. The molecules are only observed to decorate the lower part of step edges, forming one-dimensional chains. Since the [7] $\mathrm{H}$ molecules exhibit no chemical groups to form stronger directional intermolecular bonds, they do not coalesce into 2-D islands at small coverage and are imaged as unstable patterns by the STM. This mobile phase (2D gas) starts to condensate at about $50 \%$ of the saturated monolayer coverage, and short chains of [7] $\mathrm{H}$ molecules appear to nucleate randomly over the terraces. With increasing coverage enantiomorphous lattice structures embedded in areas of short-ranged ordered features are observed. Figure 4 presents STM images for the racemate at medium coverage and saturation. Overall, three pairs of enantiomorphous structures, denoted as $\varepsilon / \delta, \lambda^{\prime} / \rho^{\prime}$ and $\lambda / \rho$, are observed [37]. All these adlattices successively form with increasing global coverage and have a common feature, i.e., the molecules are aligned in zigzag double rows (see insets of Fig. 4).

The structural difference between the zigzag rows of the $\varepsilon / \delta$ and the $\lambda / \rho$ phase is, besides the slight difference in coverage (0.93 molec. $/ \mathrm{nm}^{2}$ and 0.96 molec. $\left./ \mathrm{nm}^{2}\right)$, the relative azimuthal alignment of the helices in the double rows plus the alignment of the rows relative to the substrate. Figure 5 shows the models for both structures. For the $\varepsilon / \delta$ structure the intermolecular distances within a double row are smaller, but the distance between rows is larger.

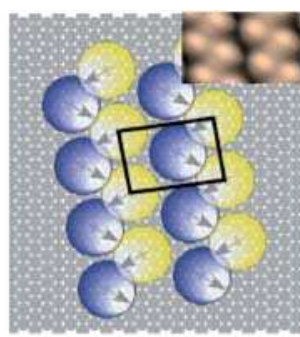

(a)

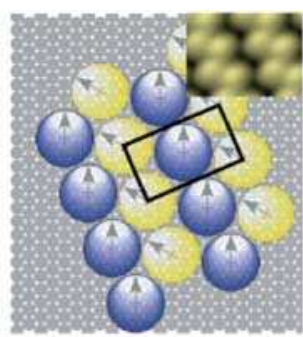

(b)

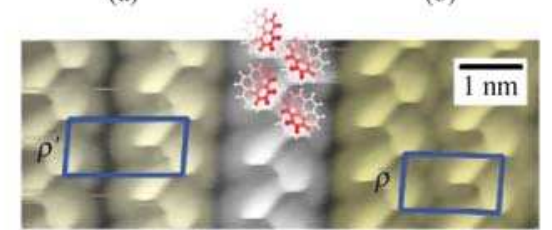

(c)

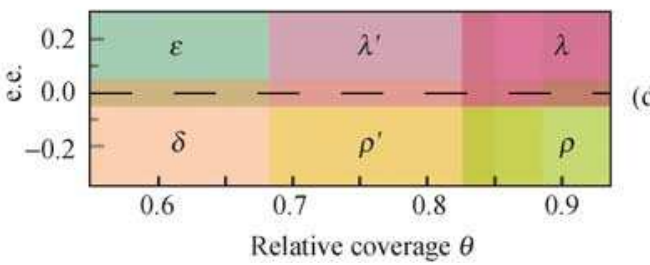

Fig. 5 (a), (b) Model for the $\delta$ domain and the $\lambda$ domain. Molecules are presented by disks. The arrows and the bright offcentre protrusions mark the overlap between the terminal rings of [7] H. (c) STM image of a phase boundary between the intermediate structure $\rho^{\prime}$ and the saturation structure $\rho$. Except the inter-double row distance, all other structural parameters are identical. (d) Phase diagram for the racemate with increasing coverage and for unequal mixtures $($ e.e. $\neq 0$ ) of the enantiomers. Small enantiomeric excess (e.e.) lets only one enantiomorph exist.

Both phases are closely packed and it comes as a surprise that the intermediate phase that appears with increasing global coverage after the $\varepsilon / \delta$ phase, has a much lower density $\left(0.77\right.$ molec. $\left./ \mathrm{nm}^{2}\right)$. The azimuthal alignment, however, is identical with the $\lambda / \rho$ structure. Only the inter-row distance is substantially larger [Fig. 5(c)].

In order to form the densest and most stable $\lambda / \rho$ packing at saturation coverage, the transition from the dense $\varepsilon / \delta$ phase goes through the loosely packed - but with respect to the final phase equally aligned $-\lambda^{\prime} / \rho^{\prime}$ phase. We must keep in mind here that only certain adsorbate sites provided by the substrate crystal and certain azimuthal orientations due to the steric interaction between the helices are allowed. This explains the more or less abrupt switch in orientation in the transition from $\varepsilon / \delta$ to $\lambda / \rho$. With increasing lateral pressure the relative azimuthal alignment of the molecules in the $\varepsilon / \delta$ phase apparently becomes destabilized. This leads to the (counterintuitive) situation that locally a less favored structure is formed [Fig. 4(a) Bottom], while globally the energy of 
the system decreases due to minimizing lateral pressure. This allows maintaining the dense $\varepsilon / \delta$ phase as part of the monolayer. The $\lambda^{\prime} / \rho^{\prime}$ phase is truly an intermediate structure. It can only coexist together with the $\varepsilon / \delta$ phase. With further increasing coverage the $\varepsilon / \delta$ phase disappears, while the $\lambda^{\prime} / \rho^{\prime}$ phase converts step-by-step into $\lambda / \rho$ domains [Fig. $5(\mathrm{~d})$ ]. A phase diagram including the structures for the pure enantiomers can be found in Ref. [37].

\subsubsection{A bowl-shaped phthalocyanine}

2-D crystallization of the subphthalocyanine (SubPc, Fig. 6, insets) reveals also interesting intermediate phases. SubPc is a special member in the family of metallic phthalocyanines because of its bowl geometry. It has only three isoindolyl groups instead of four in the common MPc. The central boron atom is $\mathrm{sp}^{3}$-coordinated to all isoindolyl groups and to the axial chlorine atom, which leads to a bowl-shaped molecular frame. SubPc is still the only known threefold symmetric phthalocyanine. SubPc adsorption and growth has been studied previously [38-42], whereby on $\mathrm{Ag}$ (111) SubPc was found to adsorb with the bowl opening pointing away from the surface. On $\mathrm{Au}(111)$ Jiang et al. recently reported an intermediate phase that, similar to the [7] $\mathrm{H} / \mathrm{Cu}(111)$ case, shows the coexistence of differently azimuthal-aligned structures. Figure 6 shows the observed structures for SubPC [43].

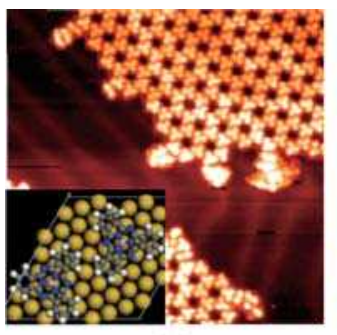

(a)

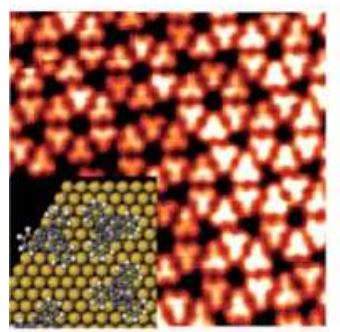

(c)

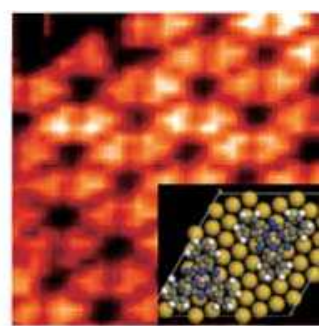

(b)

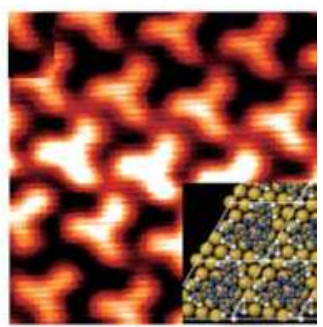

(d)
Fig. 6 STM images of different phases of subPC on Au (111) with increasing global coverage $(\theta)$. (a) $\theta=0.2,25 \mathrm{~nm} \times 25 \mathrm{~nm}$, $U=-2.0 \mathrm{~V}, I=60 \mathrm{pA}$. (b) $\theta=0.6,16 \mathrm{~nm} \times 16 \mathrm{~nm}, U=-2.0 \mathrm{~V}$, $I=100 \mathrm{pA}$. (c) $\theta=0.6,16 \mathrm{~nm} \times 16 \mathrm{~nm}, U=-2.0 \mathrm{~V}, I=$ $100 \mathrm{pA}$. (d) $\theta=1.0,5 \mathrm{~nm} \times 5 \mathrm{~nm}$. The insets show the relative alignment of the molecules in the structures.

Depending on coverage and deposition temperature, four different complex structures have been observed at $77 \mathrm{~K}$ in STM. The threefold symmetric molecule ap- pears accordingly in the STM, i.e. as three-spoke entity. The initial $(6-3,39)$ phase [Fig. 6(a), 0.032 molecules per Au atom] shows a wheel-network structure with the isoindolyl groups clockwise or counterclockwise geared to each other. As a consequence, mirror domains are found at the surface [Fig. 6(a)]. Attractive interactions dominate this structure, since island formation is observed. At higher global coverage, two coexisting phases are observed. One of the structures has identical local density as the initial $(6-3,39)$ structure, but there is no gearing in this "diamond" $(6-3,39)$ phase [43]. The relative orientation between adjacent molecules in the superlattice has changed such that the phenylene parts of two isoindolyl groups face each other [Fig. 6(b)].

The other "intermediate" structure is slightly higher in density and combines both structural features: the setoff observed for the initial phase plus the opposing phenylene rings of the diamond structure. In matrix notation the unit cell is characterized as $\left(\begin{array}{lll}13 & 0,0 & 13\end{array}\right)$ with six molecules per unit cell ( 0.036 molecules per Au atom) In contrast to the corannulene case no temperature change is involved between the equally dense phases, but there is an increase in global density. When the diamond structure nucleates and grows there are substantially more molecules on a single substrate terrace than in the case of the honeycomb formation. A limit in mobility could therefore impose a kinetic barrier, not allowing the final reorientation into a honeycomb pattern. However, like in the [7] H case, another coexisting phase with different density is present on the surface, but this time on different terraces only. Nucleation and growth of the (more stable) denser phase leads to a subcritical coverage on other terraces. Upon further cooling, the diamond phase nucleates on the other terraces with coverages below the critical value needed for intermediate phase nucleation. This mechanism is further supported by the fact that many "diamond" domains exist on a single terrace. The boundaries cannot disappear and heal, because the temperature became too low. In that respect, "diamond" and "intermediate" must coexist and both structures are actually metastable transitional phases between the lowcoverage honeycomb (ruled by attraction forces) and the saturation coverage $(4-1,15)$ phase that forms only upon annealing during deposition (0.048 molecules per $\mathrm{Au}$ atom).

\section{Conclusions}

Increasing global coverage leads to coexisting intermediate phases during nucleation and 2-D growth, which not necessarily have higher coverage than the initial phases. Our examples include different equal local density or even lower local density phases, which are in competition with other more stable phases during nucleation 
and growth. Falling temperature kinetic barriers due to lower mobility induces critical phenomena, not leading to the structure that would form without competition. Such situation is also induced by temperature-induced phase transitions with local density change. The limited mobility leads to 2-D glass and patches of low-density ordered structures. Finally, an intermediate phase that is thermodynamically stable in the temperature range between $200 \mathrm{~K}$ and $250 \mathrm{~K}$ was (meta) stabilized due to confinement with other molecules down to $60 \mathrm{~K}$. These examples show that high-resolution methods, like STM, are very valuable in order to study nucleation, growth and polymorphism, phenomena that are tremendously important for the three-dimensional outcome of materials systems.

\section{Methods and materials}

The adsorbate system has been investigated under ultrahigh vacuum (UHV) conditions $\left(p \approx 10^{-8} \mathrm{~Pa}\right)$. Corannulene, heptahelicene and subPC have been evaporated from effusion cells in vacuo onto the copper and gold crystals at room temperature. The crystal surfaces have been cleaned via standard sputtering and annealing cycles as described in detail before [44-46]. Cleanliness and quality of the surfaces and the surface coverage of the adsorbate systems have been determined with STM. Corannulene of high purity was prepared by literature methods [47]. Synthesis and enantiomeric separation (e.e. $>99.9 \%$ ) of heptahelicene $([7] \mathrm{H})$ has been performed as described previously [29, 48]. The absolute configuration was assigned with a high level of confidence by comparison of experimental and calculated VCD spectra [49]. X-ray photoelectron diffraction studies (XPD) of $(M)-[7] \mathrm{H}$ on the stepped $\mathrm{Cu}(332)$ surface confirmed this assignment [50].

Acknowledgements Support by the Schweizerischer Nationalfonds was gratefully acknowledged.

\section{References}

1. J. D. Dunitz and J. Bernstein, Acc. Chem. Res., 1995, 28: 193

2. J. Bauer, S. Spanton, R. Henry, J. Quick, W. Dziki, W. Porter, and J. Morris, Pharm. Res., 2001, 18: 859

3. R. Hilfiker (Ed.), Polymorphism in the Pharmaceutical Industry, Weinheim: Wiley-VCH, 2006

4. I. Weissbuch, L. Addadi, M. Lahav, and L. Leiserowitz, Science 1991, 253: 637

5. L. Addadi and S. Weiner, Proc. Natl. Acad. Sci., 1985, 82: 4110

6. G. Ertl, Angew. Chem. Int. Ed., 2008, 47: 3524

7. K.-H. Ernst, Top. Curr. Chem., 2006, 265: 209

8. S. De Feyter and F. De Schryver, Chem. Soc. Rev., 2003, 32: 139
9. M. J. Buerger, Elementary Crystallography: An Introduction to the Fundamental Geometrical Features of Crystals, New York: Wiley, 1956

10. D. Shechtmann, I. Blech, D. Cratias, and J. W. Cahn, Phys. Rev. Lett., 1984, 53: 1951

11. D. R. Nelson, Phys. Rev. B, 1983, 28: 5515

12. I. Hargittai (Ed.), Fivefold Symmetry, Singapore: World Scientific, 1992

13. R. Lück, Mater. Sci. Eng. A, 2000, 294-296: 263

14. K. E. Plass, A. L. Grzesiak, and A. J. Matzger, Acc. Chem. Res., 2007, 40: 287

15. T. Bauert, L. Merz, D. Bandera, M. Parschau, J. S. Siegel, and K.-H. Ernst, J. Am. Chem. Soc., 2009, 131: 3460

16. M. Parschau, R. Fasel, K.-H. Ernst, O. Gröning, L. Brandenberger, R. Schillinger, T. Greber, A. P. Seitsonen, Y. T. Wu, and J. S. Siegel, Angew. Chem. Int. Ed., 2007, 46: 8258

17. L. Merz, M. Parschau, L. Zoppi, K. K. Baldridge, J. S. Siegel, and K.-H. Ernst, Angew. Chem. Int. Ed., 2009, 48: 1966

18. L. Merz, T. Bauert, M. Parschau, G. Koller, J. S. Siegel, and K.-H. Ernst, Chem. Commun., 2009: 5871

19. L. Merz, M. Parschau, J. S. Siegel, and K.-H. Ernst, Chimia, 2009, 63: 214

20. Y. T. Wu and J. S. Siegel, Chem. Rev., 2006, 106: 4843

21. A. I. Kitajgorodskij, Acta. Cryst., 1965, 18: 585

22. J. D. Dunitz and R. M. Ibberson, Angew. Chem. Int. Ed., 2008, 474208

23. L. Merz and K.-H. Ernst, Surface Sci., 2010, 604: 1049

24. K.-H. Ernst, M. Böhringer, C. F. McFadden, P. Hug, U. Müller, and U. Ellerbeck, Nanotechnology, 1999, 10: 355

25. K.-H. Ernst, M. Neuber, M. Grunze, and U. Ellerbeck, J. Am. Chem. Soc., 2001, 123: 493

26. K.-H. Ernst, Y. Kuster, R. Fasel, C. F. McFadden, and U. Ellerbeck, Surf. Sci., 2003, 530: 195

27. R. Fasel, M. Parschau, and K.-H. Ernst, Angew. Chem. Int. Ed., 2003, 42: 5178

28. M. Parschau, U. Ellerbeck, and K.-H. Ernst, Colloids and Surfaces A: Physicochem. Eng. Aspects, 2010, 354: 240

29. K.-H. Ernst, Y. Kuster, R. Fasel, M. Müller, and U. Ellerbeck, Chirality, 2001, 13: 675

30. R. Fasel, M. Parschau, and K.-H. Ernst, Nature, 2006, 439: 449

31. M. M. Green, M. P. Reidy, R. J. Johnson, G. Darling, D. J. O'Leary, and G. Wilson, J. Am. Chem. Soc., 1989, 111: 6452

32. M. Parschau, S. Romer, and K.-H. Ernst, J. Am. Chem. Soc., 2004, 124: 15398

33. M. Parschau, T. Kampen, and K.-H. Ernst, Chem. Phys. Lett., 2005, 407: 433

34. K.-H. Ernst, Curr. Opin. Colloid Interface Sci., 2007, 13: 54

35. M. M. Green, B. A. Garetz, B Munoz, H. Chang, S. Hoke, and R. G. Cooks, J. Am. Chem. Soc., 1995, 117: 4181

36. S. Haq, N. Liu, V. Humblot, A. P. J. Jansen, and R. Raval, Nature Chem., 2009, 1: 409 
37. M. Parschau, R. Fasel, and K.-H. Ernst, Cryst. Growth \& Des., 2008, 8: 1890

38. H. Yanagi, D. Schlettwein, H. Nakayama, and T. Nishino, Phys. Rev. B, 2000, 61: 1959

39. S. Berner, M. Brunner, L. Ramoino, H. Suzuki, H.-J. Güntherodt, and T. A. Jung, Chem. Phys. Lett., 2001, 348: 175

40. S. Berner, M. de Wild, L. Ramoino, S. Ivan, A. Baratoff, H.-J. Güntherodt, H. Suzuki, and T. A. Jung, Phys. Rev. B, 2003, 68: 115410

41. H. Yanagi, K. Ikuta, H. Mukai, and T. Shibutani, Nano Lett., 2002, 2: 951

42. S. Mannsfeld, H. Reichhard, and T. Fritz, Surf. Sci., 2003, 525: 215

43. N. Jiang, Y. Wang, Q. Liu, Y. Zhang, K.-H. Ernst, and H.
J. Gao, Phys. Chem. Chem. Phys., 2010, 12: 1318

44. K.-H. Ernst, D. Schlatterbeck, and K. Christmann, Phys. Chem. Chem. Phys. 1999, 1: 4105

45. B. Behzadi, D. Ferri, A. Baiker, and K.-H. Ernst, Appl. Surf. Sci., 2007, 253: 3480

46. M. Parschau, B. Behzadi, S. Romer, and K.-H. Ernst, Surf. Interface Anal., 2006, 38: 1607

47. T. J. Seiders, E. L. Elliott, G. H. Grube, and J. S. Siegel, J. Am. Chem. Soc., 1999, 121: 7804

48. A. Sudharkar and T. J. Katz, Tetrahedron Lett., 1986, 27: 2231

49. T. Bürgi, A. Urakawa, B. Behzadi, K.-H. Ernst, and A. Baiker, New J. Chem., 2004, 28: 332

50. R. Fasel, A. Cossy, K.-H. Ernst, F. Baumberger, T. Greber, and J. Osterwalder, J. Chem. Phys., 2001, 115: 1020 